\title{
Prediction of different airway gradings in T1-2 laryngeal cancer with anterior vocal commissure involvement
}

\author{
Chunlin Luo \\ Sun Yat-sen University First Affiliated Hospital \\ Wenbin Lei ( $\square$ leiwb@mail.sysu.edu.cn ) \\ Sun Yat-sen University First Affiliated Hospital
}

\section{Research}

Keywords: modified Mallampati test, indirect laryngoscopy, anterior vocal commissure involvement, laryngeal cancer, survival

Posted Date: February 28th, 2020

DOI: https://doi.org/10.21203/rs.2.24810/v1

License: (1) (1) This work is licensed under a Creative Commons Attribution 4.0 International License. Read Full License 


\section{Abstract}

Background To investigate the clinical efficacy of the modified Mallampati test (MMT) combined with indirect laryngoscopy in measuring glottic exposure levels of early glottic laryngeal carcinoma (T1-2) with anterior vocal commissure involvement.

Methods One hundred eighty-two patients with early glottic laryngeal cancer were divided into two groups: one group comprised patients treated with transoral carbon dioxide laser microsurgery (TLM, 65 patients), and the other group comprised patients treated with open partial laryngectomy (OPL, 117 patients). The MMT and indirect laryngoscopy were used to re-evaluate the level of glottic exposure and to classify the patients based on these levels. The local control and disease-free survival rates of those patients were measured based on their MMT and indirect laryngoscopy classifications.

Results The 5-year local control rate was significantly different between the two groups according to the MMT (log-rank test: $\chi 2=4.020$, $\mathrm{P}=0.045,90.9 \%$ in the OPL group vs. $71.4 \%$ in the TLM group). For patients diagnosed with grade IV tumors using indirect laryngoscopy, the 5-year local control rate was significantly different between the two groups (log-rank test: $\chi 2=4.076, P=0.044,91.7 \%$ in the OPL group vs. $54.7 \%$ in the TLM group). Among patients diagnosed with grade III or IV tumors using indirect laryngoscopy and with class III or IV tumors using MMT, the 5-year local control rate was significantly different between the two groups (log-rank test: $\chi 2=8.037, P=0.005$, $97.14 \%$ in the OPL group vs. $75.47 \%$ in the TLM groups).

Conclusions The combination of the MMT and indirect laryngoscopy to evaluate difficult airways is relatively simple and is very useful for surgeons to better prepare for surgery.

\section{Background}

Early glottic cancer is most commonly head and neck cancer, accounting for approximately $2 \%$ of all systemic malignancies [1-3]. Transoral carbon dioxide laser microsurgery (TLM) is widely used to treat early glottic cancer [4], but its use in early glottic laryngeal cancer with anterior vocal commissure (AVC) involvement has been limited, mainly because insufficient evaluation of the range of AVC lesions could lead to recurrence [5]. Sachse et al. [6] found that the laser group and open surgery group differed in local control rates in early laryngeal cancer, which was largely due to poor glottic exposure. In 2003, the American Society of Anesthesiology (ASA) defined difficult laryngoscopy (DL) as follows: the application of conventional laryngoscopy to expose any part of the glottis, defined as difficult airway (DA). There are four manifestations of difficult airways (DAs): mask ventilation difficulties, laryngoscopic exposure difficulties, tracheal intubation difficulties and tracheal intubation failure [7]. Whether glottic fissures can be accessed under a laryngoscope has been widely investigated. At present, there are many ways to predict DA preoperatively, such as evaluating whether the patient has a short neck, mandibular retraction, loose or fixed teeth, difficulty in opening the mouth, or a cervical condition [8]; measuring the distance of hyperthyroidism; or performing the modified Mallampati test (MMT), direct laryngoscopy classification (Cormack-Lehane classification), or indirect laryngoscopy classification. The MMT is extensively used for the identification of DA, but its clinical value has been controversial [9-11]. Yamamoto et al. [12] reported that predictions using indirect laryngoscopy grading are routinely performed by otolaryngologists and are therefore more reliable than those using the MMT. In addition, this approach is convenient and fast. To date, little research has been conducted to investigate the clinical application of the Mallampati test (MMT) in combination with indirect laryngoscopy for diagnosing glottic laryngeal carcinoma (T1-2) with AVC. In this research, clinical data that were collected over the past 10 years at the First Affiliated Hospital of Sun Yat-sen University and that met the inclusion criteria were analyzed to investigate the clinical application of the MMT combined with indirect laryngoscopy in evaluating the clinical efficacy of different surgical methods on glottic laryngeal carcinoma (T1-2) with AVC. Our findings can provide guidance to physicians in preoperative assessments of patients with early glottic laryngeal cancer with AVC involvement and in choosing the appropriate treatment.

\section{Methods}

\section{General information}

\section{Study design}

Clinical data were collected from 182 patients with early glottic laryngeal carcinoma (squamous cell carcinoma confirmed pathologically) who underwent concurrent surgery at our unit between January 2006 and January 2017. Patients were divided into the TLM group (65 patients) and the open partial laryngectomy (OPL) group (117 patients). A total of 179 males and 3 females with an average age of 63.19 years and an average follow-up time of 1748.6 days (371-3939 days) were included. There were 27, 37, 83, and 26 cases of class I, II, III, and IV airways, respectively, as categorized by the MMT; 9 cases were excluded due to incomplete follow-up. For airway classification 
based on indirect laryngoscopy, there were 26, 59, 65 and 21 cases of grade I, II, III and IV airways, respectively; 11 cases were excluded due to incomplete follow-up (Table 1).

Table 1

Patient characteristics.

\begin{tabular}{|c|c|}
\hline Category & $\mathbf{N}(\%)$ \\
\hline \multicolumn{2}{|l|}{ Sex } \\
\hline Male & 179 (98.35) \\
\hline Female & $3(1.65)$ \\
\hline \multicolumn{2}{|l|}{ Age } \\
\hline$\leq 65$ & $111(60.99)$ \\
\hline$>65$ & $71(39.01)$ \\
\hline \multicolumn{2}{|c|}{ Modified Mallampati Test } \\
\hline Class I & $27(15.61)$ \\
\hline Class II & 37 (21.39) \\
\hline Class III & $83(47.98)$ \\
\hline Class IV & $26(15.03)$ \\
\hline Unknown & 9 \\
\hline \multicolumn{2}{|c|}{ Indirect Laryngoscopy } \\
\hline Grade I & $26(15.20)$ \\
\hline Grade II & $59(34.50)$ \\
\hline Grade III & 65 (38.01) \\
\hline Grade IV & $21(12.28)$ \\
\hline Unknown & 11 \\
\hline \multicolumn{2}{|c|}{$\begin{array}{l}\text { The exclusion criteria were as follows: 1) pathological confirmation of tumors other than laryngeal squamous cell carcinoma (such as } \\
\text { adenocarcinoma or papillary carcinoma); 2) clinical stage other than T1-2N0M0 (according to 2002 AJCC/UICC); 3) history of } \\
\text { oropharyngeal surgery; and 4) severe dysfunction of major organs. }\end{array}$} \\
\hline
\end{tabular}

\section{Choice of surgical methods}

\section{TLM}

Surgical equipment

A Zeiss S88 operating microscope (German Zeiss), a Sharper-30c carbon dioxide laser and coupler (Israel Medical Company), a support laryngoscope (STORZ, Germany) and a laryngeal microsurgery instrument were used.

Surgical methods

All surgeries were carried out according to the 2007 European Laryngeal Society classification for CO2 laser vocal cord resection.

OPL

The procedures included frontal laryngectomy (FLPL), partial cartilage upper laryngectomy, modified ring epiglottic bone fixation (SCPLCTP), and modified thyroid cartilage window (WPL).

\section{Research process}


All participants provided written informed consent and completed follow-up visits, including clinic visits or phone calls, for at least 12 months after surgery. In addition, outpatient follow-up was performed with the MMT and indirect laryngoscopy grading with all data recorded. This research was approved by the Ethics Committee of our University (Approval No. 2016183).

MMT method

The patient is asked to sit, place his head in a normal middle position, and open his mouth and stretch his tongue as much as possible without making any sound. The observer then looks at the patient's oropharynx and classifies the visible oropharyngeal structure. The classes are defined as class I, visible tonsillar pillars, soft palate and uvula; class II, visible tonsillar pillars and soft palate, but the uvula is blocked by the base of the tongue; class III, only the soft palate and basal part of the uvula can be seen; and Class IV: soft palate not visible. Generally, classes I and II are considered non-DAs, while classes III and IV are deemed DAs.

Indirect laryngoscopy

The airway grading is measured by indirect laryngoscopy and is graded as follows: grade I, glottis is fully visible; grade II, posterior commissure is visible; grade III, epiglottis is visible; and grade IV, no laryngeal structure is visible. Generally, grades I and II are considered non-DAs, while grades III and IV are deemed DAs.

\section{Evaluated parameters}

Local control rate, disease-free survival rate.

\section{Statistical analysis}

All data were analyzed using R3.4.3 (R Core Team, Vienna, Austria). The Kaplan-Meier method was used to estimate the survival rate at specific time points for both experimental and control groups, and the log-rank test was used to analyze the difference between the two groups. $\mathrm{P}<0.05$ indicates statistical significance.

\section{Results}

\section{Single indicator to assess the local control and disease-free survival rates of the two groups}

MMT

As shown in Tables 2 and 3 and Fig. 1, no significant difference was found between the two groups in terms of the local control rates and disease-free survival rates of class I, II, or III patients according to the MMT. The 5-year local control rate of class IV patients was significantly different between the two groups (log-rank test: $\chi 2=4.020, P=0.045$ ). The local control rates of class IV patients in the OPL and TLM groups according to the MMT were $90.9 \%$ and $71.4 \%$, respectively. The difference in disease-free survival between the two groups was similar to the difference in local control. 
Table 2

Local control rates of different airway gradings between the two treatment groups (\%).

\begin{tabular}{|c|c|c|c|c|c|c|c|c|c|c|c|c|c|}
\hline & \multirow[t]{2}{*}{ TIME } & \multicolumn{4}{|c|}{ Modified Mallampati Test } & \multicolumn{4}{|c|}{ Indirect Laryngoscopy } & \multicolumn{4}{|c|}{ United rating } \\
\hline & & $\begin{array}{l}\text { Class } \\
\text { I }\end{array}$ & $\begin{array}{l}\text { Class } \\
\text { ॥ }\end{array}$ & $\begin{array}{l}\text { Class } \\
\text { III }\end{array}$ & $\begin{array}{l}\text { Class } \\
\text { IV }\end{array}$ & $\begin{array}{l}\text { Grade } \\
\text { I }\end{array}$ & $\begin{array}{l}\text { Grade } \\
\text { II }\end{array}$ & $\begin{array}{l}\text { Grade } \\
\text { III }\end{array}$ & $\begin{array}{l}\text { Grade } \\
\text { IV }\end{array}$ & DA1 & DA2 & DA3 & DA4 \\
\hline \multirow[t]{3}{*}{ TLM } & 1 year & 100 & 100 & 94.87 & 100 & 100 & 100 & 96 & 100 & 96.77 & 100 & 100 & 100 \\
\hline & 3 year & 100 & 83.3 & 83.95 & 72.92 & 100 & 94.12 & 85.87 & 54.69 & 75.47 & 100 & 100 & 92.31 \\
\hline & 5 year & 100 & 83.3 & 83.95 & 72.92 & 100 & 94.12 & 85.87 & -* & 75.47 & 100 & - $^{*}$ & 92.31 \\
\hline \multirow[t]{3}{*}{ OPL } & 1 year & 100 & 96.55 & 100 & 100 & 100 & 100 & 97.5 & 100 & 100 & 100 & 90 & 100 \\
\hline & 3 year & 100 & 96.55 & 94.96 & 100 & 100 & 100 & 97.5 & 91.67 & 97.14 & 100 & 90 & 100 \\
\hline & 5 year & 100 & 96.55 & 94.65 & 90.91 & 100 & 96.55 & 97.5 & 91.67 & 97.14 & 91.67 & 90 & 100 \\
\hline \multicolumn{14}{|c|}{$\begin{array}{l}\text { Abbreviations: TLM, transoral carbon dioxide laser microsurgery; OPL, open partial laryngectomy. DA1 grades } 3 \text { and } 4 \text { were determined } \\
\text { via indirect laryngoscopy and modified Mallampati test classes III and IV; DA2 grades } 1 \text { and } 2 \text { were determined via indirect } \\
\text { laryngoscopy and modified Mallampati test classes III and IV; DA3 grades } 3 \text { and } 4 \text { were determined via indirect laryngoscopy and } \\
\text { modified Mallampati test classes I and II; and DA4 grades } 1 \text { and } 2 \text { were determined via indirect laryngoscopy and modified Mallampati } \\
\text { test classes I and II. }\end{array}$} \\
\hline
\end{tabular}

Table 3

Disease-free survival rates of different airway gradings between the two treatment groups (\%).

\begin{tabular}{|c|c|c|c|c|c|c|c|c|c|c|c|c|c|}
\hline & \multirow[t]{2}{*}{ TIME } & \multicolumn{4}{|c|}{ Modified Mallampati Test } & \multicolumn{4}{|c|}{ Indirect Laryngoscopy } & \multicolumn{4}{|c|}{ United Rating } \\
\hline & & Class & $\begin{array}{l}\text { Class } \\
\text { II }\end{array}$ & $\begin{array}{l}\text { Class } \\
\text { III }\end{array}$ & $\begin{array}{l}\text { Class } \\
\text { IV }\end{array}$ & $\begin{array}{l}\text { Grade } \\
\text { I }\end{array}$ & $\begin{array}{l}\text { Grade } \\
\text { II }\end{array}$ & $\begin{array}{l}\text { Grade } \\
\text { III }\end{array}$ & $\begin{array}{l}\text { Grade } \\
\text { IV }\end{array}$ & DA1 & DA2 & DA3 & DA4 \\
\hline \multirow[t]{3}{*}{ TLM } & 1 year & 100 & 97.37 & 100 & 100 & 100 & 100 & 96 & 100 & 96.77 & 100 & 100 & 100 \\
\hline & 3 year & 92.86 & 86.16 & 72.92 & 72.92 & 100 & 94.12 & 85.87 & 54.69 & 75.47 & 100 & 100 & 92.31 \\
\hline & 5 year & 92.86 & 86.16 & 72.92 & 72.92 & 100 & 94.12 & 85.87 & $-*$ & 75.47 & 100 & $-*$ & 92.31 \\
\hline \multirow[t]{3}{*}{ OPL } & 1 year & 97.92 & 100 & 100 & 100 & 100 & 100 & 97.5 & 100 & 100 & 100 & 90 & 100 \\
\hline & 3 year & 97.92 & 92.46 & 94.12 & 100 & 100 & 100 & 97.5 & 91.67 & 97.14 & 100 & 90 & 100 \\
\hline & 5 year & 97.92 & 92.46 & 85.56 & 90.91 & 100 & 96.55 & 97.5 & 91.67 & 97.14 & 91.67 & 90 & 100 \\
\hline \multicolumn{14}{|c|}{$\begin{array}{l}\text { Abbreviations: TLM, transoral carbon dioxide laser microsurgery; OPL, open partial laryngectomy. DA1 grades } 3 \text { and } 4 \text { were determined } \\
\text { via indirect laryngoscopy and modified Mallampati test classes III and IV; DA2 grades } 1 \text { and } 2 \text { were determined via indirect } \\
\text { laryngoscopy and modified Mallampati test classes III and IV; DA3 grades } 3 \text { and } 4 \text { were determined via indirect laryngoscopy and } \\
\text { modified Mallampati test classes I and II; and DA4 grades } 1 \text { and } 2 \text { were determined via indirect laryngoscopy and modified Mallampati } \\
\text { test classes I and II. }\end{array}$} \\
\hline
\end{tabular}

a Follow-up time of the study subjects did not reach the corresponding time.

\section{Indirect laryngoscopy}

As shown in Tables 2 and 3 and Fig. 2, there was no significant difference in the local control and disease-free survival rates between the two groups among grade I, II, and III patients according to indirect laryngoscopy at 5 years. However, the 5-year local control rates of patients with grade IV airways (indirect laryngoscopy) were significantly different between the OPL and TLM groups (log-rank test: $\chi 2$ = $4.076, P=0.044$ ), with 5-year local control rates of $91.7 \%$ and $54.7 \%$, respectively. The same pattern was observed for disease-free survival rates between the two groups.

\section{Local control rate and disease-free survival rate based on a combination of indicators}


As shown in Tables 2 and 3 and Fig. 3, when indirect laryngoscopy was combined with the MMT, there was no significant difference in the local control and disease-free survival rates at 5 years between class I/II and grade I/II patients. However, when grade III or IV patients according to indirect laryngoscopy were combined with class III or IV patients according to the MMT, the 5-year local control rate was significantly different between the OPL and TLM groups ( $97.14 \%$ vs. $75.47 \%$, respectively; log-rank test: $\chi 2=8.037, P=0.005)$. The results for disease-free survival rate were similar.

In the TLM group, when grade III or IV patients according to indirect laryngoscopy were combined with class III or IV patients according to the MMT, the 5-year local control rate was significantly different between the OPL and TLM groups $(97.14 \%$ vs. $75.5 \%$, respectively; logrank test: $\chi 2=4.028, P=0.045)$.

\section{Correlation between indirect laryngoscopy and the MMT}

Data analysis showed that there was a moderate correlation between indirect laryngoscopy and the MMT $(r=0.646, P<0.05)$.

\section{Discussion}

It has been reported that the incidence of DA is $6.1 \%-10.1 \%$. $[13,14]$ As shown in Table 1, the incidence of difficult airways was slightly higher for MMT grade IV patients $(26 / 182,15.03 \%)$ and indirect laryngoscopy grade IV patients $(21 / 182,12.28 \%)$, whereas in Tables 2 and 3 , no difference between MMT grade III and indirect laryngoscopy grade III patients was found in terms of DAs. If the patient has obvious anatomical or pathological abnormalities of the airway, it is necessary to determine whether DA exists before further surgery. Predictive studies are clinically significant when the structures are normal, but other factors may make glottis exposure difficult. To predict whether an airway is difficult, either a single factor model or a combination of two or more factors should be used.

MMT

MMT is widely used in DA diagnosis. In 1983, Khan et al. [15] proposed a method to classify DAs into three levels according to larynx structure. Nasir et al. [16] expanded this classification to four levels, which drew much interest among researchers, but its clinical practice has been questioned since its classification was introduced. The MMT is a comprehensive index that is mainly based on mouth opening width, tongue size and mobility, maxillary movement, other intraoral structures and cranial and cervical joint movements. The grading of glossopharyngeal structure is closely related to the ease of endotracheal intubation under direct laryngoscopy, so it has remained the primary assessment method for anesthesiologists. Adamus et al. [17] reported that the specificity and sensitivity of the MMT are not high, limiting its clinical use. In addition, some scholars believe that its accuracy is moderately low $[18,19]$. Other reports, including metaanalyses, have shown divergent results. Lee et al. [20] suggested that the MMT should not be used as the sole assessment tool, a view shared by most scholars. When the MMT is used alone, there is no way to fully confirm the DA. Stud have shown that the combination of the MMT with hyperthyroidism or other airway assessments can improve the diagnostic accuracy up to $50-80 \%[10,21]$. El-Ganzouri et al. [22] analyzed 10507 cases, with MMT class III as the boundary value, and defined Cormack-Lehane grades 3 and 4 as tracheal intubation difficulties. The sensitivity of the MMT in predicting tracheal intubation difficulty was $44.7 \%$, while its specificity was $89.0 \%$, with a positive predictive value of $21 \%$ and a negative predictive value of $96.1 \%$ [23]. From this perspective, the MMT has clinical value. Since the current study was a retrospective analysis in nature and did not obtain the number of specific difficult exposures during surgery, the relevant sensitivity, specificity and other indicators were not evaluated. However, our research did show a statistically significant difference between the two groups among class IV patients according to the MMT, indicating that although a class III or IV classification according to the MMT is defined as a DA, the score may not be sufficient in the single factor analysis. The results of this study suggest that the MMT is a more accurate predictor of DA among class IV patients.

\section{Indirect laryngoscopy}

The indirect laryngoscope was invented in 1854 by Spanish vocal teacher Manuel Garcia, who became the first person to observe his own throat structure. His design was subsequently improved by Professor Ludwig Turck, while the predecessor of modern laryngoscope was designed by Johann Nepomuk Gzermak in Poland in 1858. This technology has been rapidly utilized as one of the most common and simple inspection instruments in the otolaryngology field. In 1997, Yamamoto et al. [12] first described airway grading using indirect laryngoscopy as follows: grade I, the entire glottis is visible; grade II, the posterior commissure is visible; grade III, only the epiglottis is visible; and grade IV, no laryngeal structures are visible. Generally, grade III or above is indicative of DA. Sanchez-Morillo et al. [23] found that indirect laryngoscopy was an independent and reliable tool to diagnose DAs when combined with $70^{\circ}$ rigid endoscopy and direct laryngoscopy. Additionally, Yamamoto et al. [12] found that the indirect laryngoscopy classification is more accurate than the direct laryngoscopy prediction, indicating that indirect laryngoscopy alone has certain clinical significance. Therefore, the use of indirect 
laryngoscopy alone was assessed in this study. When the indirect laryngoscopy result was grade IV, the two treatment groups showed statistically significant differences in both 5-year local control and disease-free survival rates. Additionally, the 5-year local control and disease-free survival rates based on indirect laryngoscopy were lower than those based on the MMT, indicating that indirect laryngoscopy grade IV could better diagnose DA and lead to higher diagnosis accuracy, which is consistent with the results from previous reports.

\section{Combination of the MMT and indirect laryngoscopy to evaluate DAs}

In clinical practice, the combination of multiple methods can diagnose DAs more accurately and reliably. For example, Merah et al. [21] combined the MMT, intercondylar distance, chest-to-twist distance, mandible length and upper and lower incisor distance to diagnose DAs. The joint diagnostic value of this combined method was the highest, but multiple shortcomings limited the application of this method. One of the shortcomings is that this combined approach is not easy to implement in practice. Therefore, our study was designed to elucidate DA patients with different exposure levels based on two assessment tools and to analyze the survival rate of patients with laryngeal cancer with AVC based on these tools. Our research found that patients with DAs (class III/IV) who underwent TLM had a lower local control rate than those who underwent OPL. Because this difference was statistically significant, combining the MMT with indirect laryngoscopy can reflect to some extent the exposure difficulties that affect prognosis. This study has clinical significance when the anatomy looks normal, but there may be difficulties in glottic exposure. After all, patients with MMT grade III or indirect laryngoscopy class III airways account for the majority of patients with laryngeal cancer with AVC. Our research showed that class III/IV and grade III/IV classifications are more meaningful when combined. In addition, these two methods are relatively simple to implement and should be used more often in clinical practice.

\section{Strategy for difficult glottic exposure}

Ohno et al. [24] reported that laryngoscopy and body posture are important for AVC exposure. Some scholars have found that assessing glottic exposure requires information on sex and thyroid mandibular angle (TMA). If the patient is female and the TMA is greater than $120^{\circ}$, the positive local control rate can reach as high as $94.6 \%[25,26]$. For patients who have early glottic laryngeal cancer with AVC involvement and undergo laser surgery, the AVC exposure field requirements are higher. In addition to adequate preoperative assessment, surgical techniques and surgeon experience also affect surgical outcomes [27]. For example, in cases of poor glottic exposure, the smaller STORZ mirror can be replaced with a more rigid endoscope $\left(30^{\circ}, 45^{\circ}\right.$, or $\left.70^{\circ}\right)$ to fully expose the lesion [28]. If the target area is still insufficiently exposed, the thyroid cartilage can be pressed to shift the vocal cords before initial exposure or after the first $1 / 3$ of the area is exposed. The goal is to remove the lesion under direct vision during surgery. If the MMT or indirect laryngoscopy is used alone, although there is a certain correlation between these classifications and DAs, surgeons should be aware of the possibility of false negative results and the inability to diagnose DAs or the possibility of unnecessary treatment when false positive results occur. It is important to balance the pros and cons and make optimal decisions for patients. For laryngeal cancer with AVC involvement, whether it is DA or another impeding factor, it is necessary to comprehensively evaluate all aspects. However, there is no indicator or method that can be used to identify appropriate exposure. Therefore, if there is still poor exposure after trying different approaches during surgery, the surgeon must be willing to adjust the treatment strategies in a timely manner.

\section{Research Limitations}

Since this was a retrospective study, not a prospective study, there were some missing data regarding related factors, which may lead to false positive results. Second, further follow-up and long-term comparison of the local control and disease-free survival rates of TLM and OPL groups are needed. Additional postoperative patient survival data, including MMT scores, upper and lower incisor spacing, hyperthyroidism spacing, mandibular advancement amplitude, head and neck movement amplitude, laryngoscopic exposure grade and $\mathrm{BMI}$, need to be further analyzed.

\section{Conclusions}

For T1-2 laryngeal cancer with AVC involvement, patients with preoperative class IV airway according to the MMT, grade IV according to indirect laryngoscopy or a combination of class III/IV and grade III/IV are more likely to have DAs. Surgeons need to prepare accordingly with intraoperative response measures and the potential shift to open surgery at any time. The combination of the MMT and indirect laryngoscopy to evaluate DAs is relatively simple and can be used in clinical practice.

\section{Abbreviations}

TLM=transoral carbon dioxide laser microsurgery, OPL=open partial laryngectomy, LC=local control, DFS=disease-free survival, OS=overall survival, LFP=laryngeal function preservation, AVC=anterior vocal commissure, FLPL=frontolateral partial laryngectomy, SCPL- 
CTP=supracricoid partial laryngectomy with cricothyroidopexy, WPL=window partial laryngectomy.

\section{Declarations}

\section{Ethics approval and consent to participate}

This research was approved by the Ethics Committee of The First Affiliated Hospital of Sun Yat-sen University.

Approved No. of ethic committee:2016183.

\section{Consent for publication}

Not applicable

\section{Availability of data and materials}

All data generated or analyzed during this study are included in this published article [and the supplementary information files].

\section{Competing interests}

The authors declare that they have no competing interests.

\section{Funding}

This work was supported by the Guangzhou Science and Technology Program (201605030003), the 5010 Clinical Research Program of Sun Yat-sen University, the Natural Science Foundation of Guangdong Province of China (2016A030313247), the Science and Technology Program of Guangzhou of China (201704020092), and the Science and Technology Planning Project of Guangdong Province of China (2017A050506018). These funds had played an important role in the design of the study and collection, analysis, and interpretation of data and in writing the manuscript.

\section{Authors' contributions}

LC collected date, drafted the work and revised the article; LW made contributions to revise article and design the study.

\section{Acknowledgements}

Not applicable

\section{Authors' information}

Chunlin Luo, Affiliation: The First Affiliated Hospital, Sun Yat-sen University. Hui Ya Hospital of The First Affiliated Hospital, Sun Yat-sen University; Email: alex3512006@126.com.

Wenbin Lei, corresponding author

The First Affiliated Hospital, Sun Yat-sen University, Guangzhou, Guangdong Province 510080, China

Email: leiwb@mail.sysu.edu.cn. 


\section{References}

1. Burch JD, Howe GR, Miller AB, Semenciw R. Tobacco, alcohol, asbestos, and nickel in the etiology of cancer of the larynx: a casecontrol study. J Natl Cancer Inst. 1981;67:1219-24.

2. Silverberg E, Boring CC, Squires TS. Cancer statistics, 1990. CA: Cancer J Clin. 1990;40:9-26.

3. Marshak G, Brenner B, Shvero J, Shapira J, Ophir D, Hochman I, et al. Prognostic factors for local control of early glottic cancer: the Rabin Medical Center retrospective study on 207 patients. Int J Radiat Oncol Biol Phys. 1999;43:1009-13.

4. de Gier HHW, Knegt PPM, de Boer MF, Meeuwis CA, van der Velden L-A, Kerrebijn JDF. CO2-laser treatment of recurrent glottic carcinoma. Head Neck. 2001;23:177-80.

5. Back G, Sood S. The management of early laryngeal cancer: options for patients and therapists. Curr Opin Otolaryngol Head Neck Surg. 2005;13:85-91.

6. Sachse F, Stoll W, Rudack C. Evaluation of treatment results with regard to initial anterior commissure involvement in early glottic carcinoma treated by external partial surgery or transoral laser microresection. Head Neck. 2009;31:531-7.

7. American Society of Anesthesiologists Task Force on Management of the Difficult Airway. Practice guidelines for management of the difficult airway: an updated report by the American Society of Anesthesiologists Task Force on Management of the Difficult Airway. Anesthesiology. 2003;98:1269-77.

8. Hartl DM, Brasnu DF. Contemporary surgical management of early glottic cancer. Otolaryngol Clin North Am. 2015;48:611-25.

9. Cattano D, Panicucci E, Paolicchi A, Forfori F, Giunta F, Hagberg C. Risk factors assessment of the difficult airway: an Italian survey of 1956 patients. Anesth Analg. 2004:1774-9.

10. Shiga T, Wajima Zi, Inoue T, Sakamoto A. Predicting difficult intubation in apparently normal patients: a meta-analysis of bedside screening test performance. Anesthesiology. 2005;103:429-37.

11. Karkouti K, Rose DK, Wigglesworth D, Cohen MM. Predicting difficult intubation: a multivariable analysis. Can J Anaesth. 2000;47:7309.

12. Yamamoto K, Tsubokawa T, Shibata K, Ohmura S, Nitta S, Kobayashi T. Predicting difficult intubation with indirect laryngoscopy. Anesthesiology. 1997;86:316-21.

13. Lundstrøm LH, Vester-Andersen M, Møller AM, Charuluxananan S, L'Hermite J, Wetterslev J. Poor prognostic value of the modified Mallampati score: a meta-analysis involving 177088 patients. Br J Anaesth. 2011;107:659-67.

14. Patil VU, Stehling LC, Zauder HL. Fiberoptic endoscopy in anesthesia. Chicago: Year Book Medical Publishers Inc.; 1983.

15. Khan ZH, Kashfi A, Ebrahimkhani E. A comparison of the upper lip bite test (a simple new technique) with modified Mallampati classification in predicting difficulty in endotracheal intubation: a prospective blinded study. Anesth Analg. 2003;96:595-9.

16. Nasir K, Shahani A, Maqbool M. Correlative value of airway assessment by Mallampati classification and Cormack and Lehane grading. Rawal Med J. 2011;36:2-6.

17. Adamus M, Fritscherova S, Hrabalek L, Gabrhelik T, Zapletalova J, Janout V. Mallampati test as a predictor of laryngoscopic view. Biomed Pap Med Fac Univ Palacky Olomouc Czech Repub. 2010;154:339-43.

18. Karkouti K, Rose DK, Ferris LE, Wigglesworth DF, Meisami-Fard T, Lee H. Inter-observer reliability of ten tests used for predicting difficult tracheal intubation. Can J Anaesth. 1996;43:554-9.

19. Tham EJ, Gildersleve CD, Sanders LD, Mapleson WW, Vaughan RS. Effects of posture, phonation and observer on Mallampati classification. Br J Anaesth. 1992;68:32-8.

20. Lee A, Fan LTY, Gin T, Karmakar MK, Ngan Kee WD. A systematic review (meta-analysis) of the accuracy of the Mallampati tests to predict the difficult airway. Anesth Analg. 2006;102:1867-78.

21. Merah NA, Wong DT, Ffoulkes-Crabbe DJ, Kushimo OT, Bode CO. Cardiothoracic anesthesia, respiration and airway modified mallampati test, thyromental distance and inter-incisor gap are the best predictors of difficult laryngoscopy in West Africans. Can $\mathrm{J}$ Anaesth. 2005;52:291-6.

22. El-Ganzouri AR, McCarthy RJ, Tuman KJ, Tanck EN, Ivankovich AD. Preoperative airway assessment: predictive value of a multivariate risk index. Anesth Analg. 1996;82:1197-204.

23. Sanchez-Morillo J, Estruch-Pérez MJ, Hernández-Cádiz MJ, Tamarit-Conejeros JM, Gómez-Diago L, Richart-Aznar M. Indirect laryngoscopy with rigid 70-degree laryngoscope as a predictor of difficult direct laryngoscopy. Acta Otorrinolaringol (Engl Ed). 2012;63:272-9. 
24. Ohno S, Hirano S, Tateya I, Kojima T, Ito J. Management of vocal fold lesions in difficult laryngeal exposure patients in phonomicrosurgery. Auris Nasus Larynx. 2011;38:373-80.

25. Bajaj Y, Uppal S, Sharma RK, Grace ARH, Howard DM, Nicolaides AR, et al. Evaluation of voice and quality of life after transoral endoscopic laser resection of early glottic carcinoma. J Laryngol Otol. 2011;125:706-13.

26. Hsiung M-W, Pai L, Kang B-H, Wang B-L, Wong C-S, Wang H-W. Clinical predictors of difficult laryngeal exposure. Laryngoscope. 2004;114:358-63.

27. Herranz J, Gavilán J, Vázquez-Barros JC. Carcinoma de comisura anterior. Acta Otorrinolaringol Esp. 2007;58:367-70.

28. Galli A, Giordano L, Sarandria D, di Santo D, Bussi M. Oncological and complication assessment of CO2 laser-assisted endoscopic surgery for T1-T2 glottic tumours: clinical experience. Acta Otorhinolaryngol Ital. 2016;36:167-73.

\section{Figures}

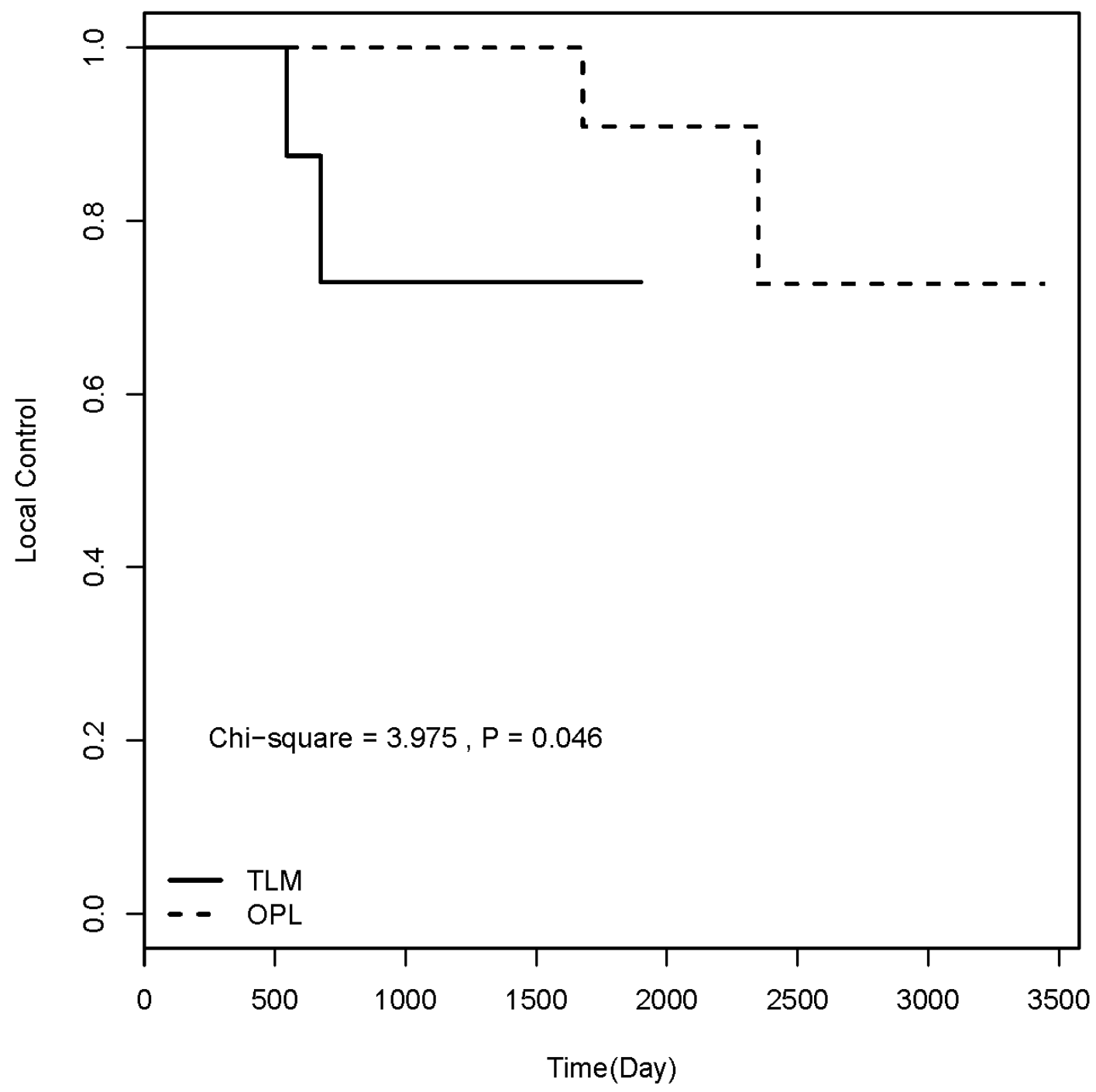

Figure 1

Comparison of survival curves between the two treatment groups among class IV patients according to the modified Mallampati test. Analysis: Comparison of the local control rate after laser surgery (TLM, solid line) and open surgery (OPL, dashed line) using Kaplan-Meier analysis. The differences were statistically significant at years 1, 3, and 5 after surgery (log-rank test $=3.975, P=0.046$ ). 


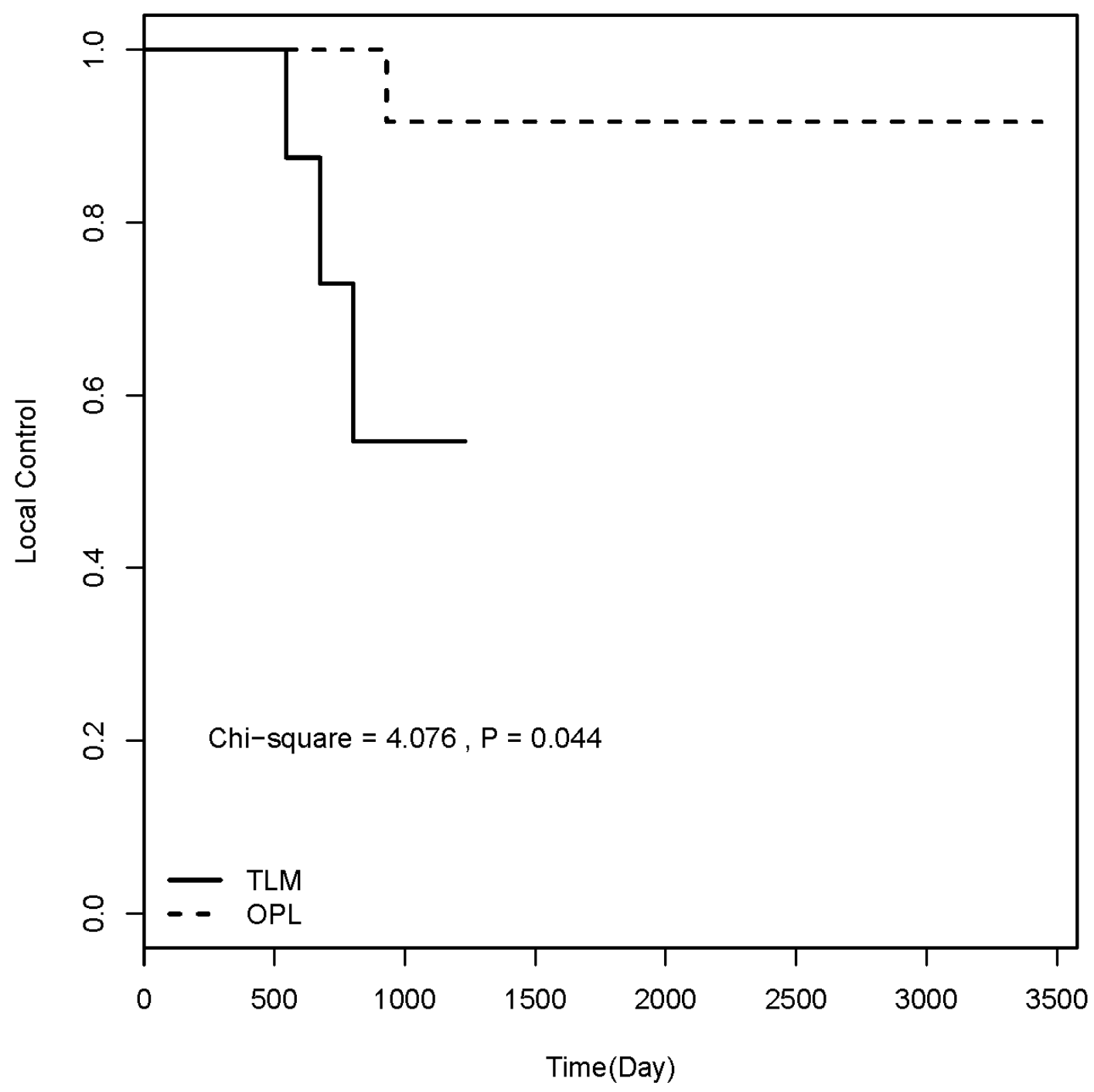

\section{Figure 2}

Comparison of survival curves between the two treatment groups among the grade IV patients according to indirect laryngoscopy. Analysis: Comparison of the local control rate after laser surgery (TLM, solid line) and open surgery (OPL, dashed line) using Kaplan-Meier analysis. The differences were statistically significant at 1,3 , and 5 years after surgery (log-rank test $=4.076, P=0.044$ ). 


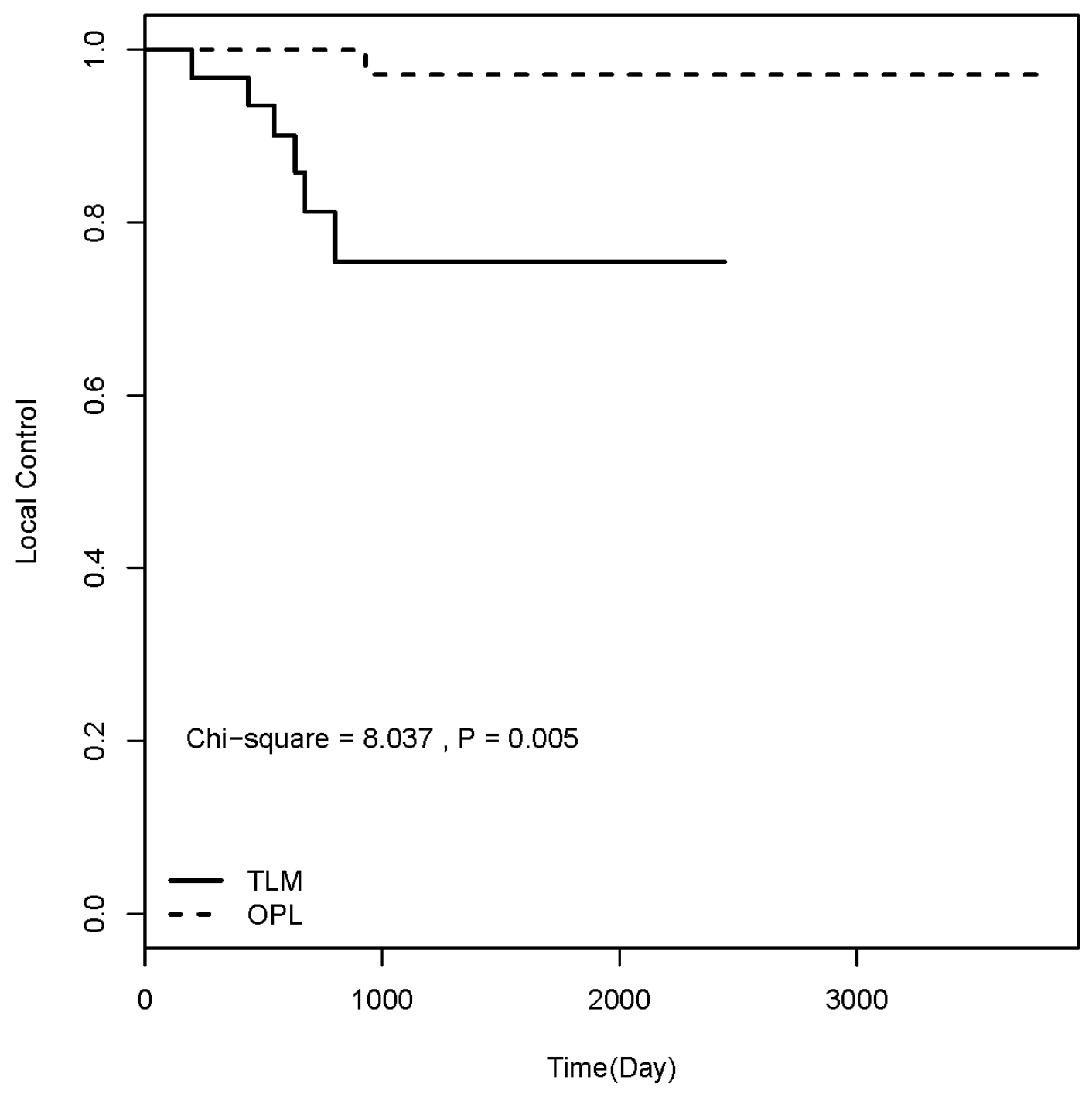

\section{Figure 3}

Comparison of the survival curves of the two groups in the combined two-group rating. Analysis: Comparison of the local control rate after laser surgery (TLM, solid line) and open surgery (OPL, dashed line) in class III and IV patients according to the MMT and those with indirect laryngoscopy grades III and IV using the Kaplan-Meier method. Differences were statistically significant (Log-rank test=8.037, P=0.005).

\section{Supplementary Files}

This is a list of supplementary files associated with this preprint. Click to download.

- redate.xlsx 